Background: Juvenile dermatomyositis (JDM) is a systemic, autoimmune inflammatory muscle disorder and vasculopathy that affects children younger than 18 years. Although the cause of JDM remains unknown it is clear that genetic and environmental influences play a role in the aetiology. New treatments are becoming available and being tested through international multicentre trials. Increasing evidence suggests a role for types I and II IFN in juvenile and adult dermatomyositis, including elevated IFN-response gene signatures in the muscle, skin and blood. It has recently been reported that patients with refractory JDM responded well to treatment with tofacitinib, a JAK inhibitor, with corresponding downregulation in selected IFN-response genes.

Objectives: In this study, we evaluated our cases with resistant JDM who received tofacitinib treatment.

Methods: Six patients who received tofacitinib because of severe skin involvement of JDM were included in the study. The data were obtained retrospectively from the hospital records.

Results: The age ranges of the cases were between 7-17 years and the ratio of girls and boys was $1(3 / 3)$. The age of diagnosis was between $2-13$ years, and the follow-up period was between 3-9 years. Calcinosis cutis in 5 cases, decreased muscle strength in 3 cases, joint involvement in 4 cases were detected.. Systemic steroids, methotrexate, and non-steroid anti-inflammatory drugs were given in all cases before tofacitinib treatment. Pamidronate was used in 4 cases because of severe skin calcinosis, high dose intravenous immunoglobulin in 4 cases, mycophenolate mofetil in 3 cases, rituximab in 3 cases and cyclophosphamide in 1 case previously. Tofacitinib treatment (10mg/gün) was started in six cases with treatment-resistant JDM. Five cases had been treated with tofacitinib for 6-24 month intervals. The treatment was discontinued in one case because of severe allergic reaction. Variable level of improvement were detected in the skin findings of all cases during the therapy period. The treatment was interrupted for 1 month in only one case due to neutropenia.

Conclusion: Tofacitinib seems to be an effective and safe treatment option in patients with JDM who are resistant to conventional treatments. More studies are needed on this subject.

Disclosure of Interests: None declared

DOI: 10.1136/annrheumdis-2020-eular.6022

\section{FRI0460 $\quad$ SAFETY OF BIOLOGICAL AGENTS IN JUVENILE IDIOPATHIC ARTHRITIS: A META-ANALYSIS OF OBSERVATIONAL STUDIES}

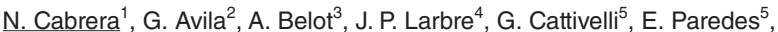
B. Kassai ${ }^{6}$, R. Euvrard ${ }^{7}$, G. Grenet ${ }^{1}$, A. Berard ${ }^{1}$, S. Mainbourg ${ }^{7}$, J. C. Lega ${ }^{7}$. ${ }^{1}$ University Lyon 1, Laboratoire de Biométrie et Biologie Évolutive, Lyon, France; ${ }^{2}$ Universidad Nacional de Asunción, Department of Research, San Lorenzo, Paraguay; ${ }^{3}$ Hospital Woman Mère Enfant - HCL, Department of Paediatric Rheumatology, Bron, France; ${ }^{4}$ Lyon Sud Hospital Center, Department of Rheumatology, Pierre-Bénite, France; ${ }^{5}$ Universidad Nacional de Asunción, San Lorenzo, Paraguay; ${ }^{6}$ University Lyon 1, Department of Pharmaco-Toxicology, Lyon, France; ${ }^{7}$ Lyon Sud Hospital Center, Department of Internal and Vascular Medicine, Pierre-Bénite, France

Background: Follow-up cohorts (observational studies) were initiated consecutively or simultaneously to the development of randomised controlled trials (RCTs) in JIA patients ${ }^{(1,2)}$. They help to identify many complications observed only in clinical practice related to off label use, coadministration of treatments, drug misuse, and occurrence of rare or unexpected event. In addition, observational studies include a higher number of patients with a longer duration of follow-up compared to randomised trials. Hence, they have a higher power to capture the occurrence of serious adverse events (SAE) in daily clinical practice ${ }^{3}$.

Objectives: To estimate the incidence of serious adverse events (SAEs) including serious infections, malignancies, and death in patients with juvenile idiopathic arthritis (JIA) treated with biological agents (BAs) in daily clinical practice, using meta-analysis techniques.

Methods: We systematically searched, up to May 2019, Medline and Embase databases for observational studies performed in JIA disease under BAs treatment. Outcomes were SAEs, serious infections, malignancies and all-cause mortality. Complementary, the incidence of SAEs in randomised controlled trials (RCTs) with withdrawal and parallel designs was performed by meta-analysis. Results: A total of 31 observational studies were included (6811 patients totalizing 17530 patients-years [PY] of follow-up). The incidence rate of SAEs was similar in observational cohorts and withdrawal RCTs (4.46 events per $100 \mathrm{PY}$, $\left.95 \% \mathrm{Cl} 2.85-6.38, \mathrm{I}^{2}=95 \%\right)$ and 3.71 events per $100 \mathrm{PY}(95 \% \mathrm{Cl} 0.0-13.34)$, $\mathrm{I}^{2}=56 \%$, respectively). The incidence of SAE was lower in parallel RCT. The incidence rate of serious infections, malignancies and death in observationa cohorts was estimated at 0.74 events per $100 \mathrm{PY}\left(95 \% \mathrm{Cl} 0.32-1.30, \mathrm{I}^{2}=83 \%\right)$, 0.10 events per $100 \mathrm{PY}\left(95 \% \mathrm{Cl} 0.06-0.16, \mathrm{I}^{2}=0 \%\right)$ and 0.09 events per 100 PY (95\% Cl 0.05-0.14, $\mathrm{I}^{2}=0 \%$ ), respectively. Infections were the known cause of death in 8 of the 14 deaths. In meta-regression and subgroup analysis, variation of serious infections rates were partially explained by follow-up time $\left(R^{2}=30.3 \%\right.$, $p=0.0008$ ), JIA categories (all JIA versus polyarticular versus systemic JIA categories, $p=0.001$ ) and cohort quality (Newcastle-Ottawa score $\geq$ to 6 versus $\leq$ to 5 stars, $p=0.0025$ ).

Conclusion: Our results suggest that the incidence rate of SAEs related to BAs in JIA disease is similar to those observed in randomised withdrawal trials. The overall incidence remained low. However, unsatisfactory description of SAEs prevents analysis of hospitalisation causes. Infection and, to a lesser extent, cancer and death, explain only part of burden of BAs.

References:

[1] Berard RA, Laxer RM. Learning the hard way: clinical trials in juvenile idiopathic arthritis. Ann Rheum Dis. 2018;77(1):1-2.

[2] Swart J, Giancane G, Horneff G, Magnusson B, Hofer M, Alexeeva E, et al. Pharmacovigilance in juvenile idiopathic arthritis patients treated with biologic or synthetic drugs: combined data of more than 15,000 patients from Pharmachild and national registries. Arthritis Res Ther. 2018 27:20(1):285.

[3] Monti S, Grosso V, Todoerti M, Caporali R. Randomized controlled trials and real-world data: differences and similarities to untangle literature data. Rheumatol Oxf Engl. 2018 01;57(57 Suppl 7):vii54-

Disclosure of Interests: None declared

DOI: 10.1136/annrheumdis-2020-eular.6433

\section{FRI0461 THE ANTI-VACCINE ANTIBODY AGAINST MEASLES, PAROTITIS, RUBELLA, DIPHTHERIA AND HEPATITIS B IN 170 JUVENILE IDIOPATHIC ARTHRITIS PATIENTS}

N. Lybimova ${ }^{1}$, I. Fridman ${ }^{2}$, O. Goleva ${ }^{2}$, R. Raupov ${ }^{3}$, M. Kaneva ${ }^{3}$, S. Kharit ${ }^{2,3}$ M. Kostik ${ }^{1,3}$. 'Almazov National Medical Research Centre, Saint Petersburg, Russian Federation; ${ }^{2}$ Pediatric Research and Clinical Center for Infection Diseases, Saint-Petersburg, Russian Federation; ${ }^{3}$ Saint-Petersburg State Pediatric Medical University, Saint Petersburg, Russian Federation

Background: Patients with juvenile idiopathic arthritis (JIA) may have lower protective levels of anti-vaccine antibodies due to high inflammatory activity, interrupted or incomplete vaccination schedule, and due to using of immune-modulating drugs, e.g. systemic corticosteroids (CS), methotrexate (MTX) and biologics.

Objectives: The aim of our study was to find the predictors of low levels of anti-vaccine antibodies in patients with JIA.

Methods: In the present study were included data 170 JIA (55 boys and 115 girls) aged from 2 to 17 years, who received scheduled vaccination before the age of 2 years and before JIA onset against measles, parotitis, hepatitis $B$, diphtheria and rubella. In all patients the $\lg \mathrm{G}$ anti-vaccine antibodies levels were detected with ELISA. In each patient we evaluate the type of the disease (oligoarthritis - 73, polyarthritis - 61 , systemic-16 and enthesitis-related arthritis -20), onset age, presence of uveitis, duration of JIA, treatment with corticosteroids (CS), methotrexate (MTX) and biologics. Data presented with median and $25 \%-75 \%$.

Results: The main demographic characteristics: age of inclusion in the study 11.4 (7.6-14.8) years, disease onset - $6.0(3.7-9.0)$ years, disease duration -3.8 (1.9-6.5) years. Treatment with CS was in 43 (25.3\%), MTX in $154(90,6 \%)$ and biologics $82(48.2 \%)$ patients, among them 53 had TNFa-inhibitors. More than 1 biologic consequently received 16/82 (19.5\%) patients. Protective levels of anti-measles antibodies was in $98(57,6 \%)$ of all JIA population, anti-parotitis $136(80.0 \%)$, anti-hepatitis B - 85 (50.0\%), anti-diphtheria - $88(51,7 \%)$, anti-rubella $-167(98.8 \%)$. Data of vaccination status and anti-vaccine antibodies levels in the table. In univariate and multivariate regression analysis the main risk factors for anti-measles antibodies levels were MTX using $(p=0.045)$, more than 1 biologics $(p=0.0004)$; for anti-hepatitis $B-M T X(p=0.03)$, for anti-diphtheria antibodies: onset age ( $p=0.0002)$, JIA duration ( $p=0.00007)$, number vaccine doses $(p=0.02)$, more than 1 biologics $(p=0.01)$; combined treatment with biologics and other drugs (MTX or CS). 


\begin{tabular}{|c|c|c|c|c|}
\hline Parameter & $\begin{array}{l}\text { No treatment } \\
\quad(n=14)\end{array}$ & $\begin{array}{l}\text { MTT, only } \\
(\mathrm{n}=74)\end{array}$ & $\begin{array}{l}\text { Biologics } \pm M T T \\
\pm C S(n=82)\end{array}$ & $P$ \\
\hline $\begin{array}{l}\text { \# anti-measels vaccine } \\
\text { doses }\end{array}$ & $2.0(2.0 ; 2.0)$ & $2.0(1.0 ; 2.0)$ & $2.0(1.0 ; 2.0)$ & 0.19 \\
\hline Anti-measels lgG, Me/ml & $0.28(0.1 ; 0.6)$ & $0.4(0.1-0.7)$ & $0.17(0.0 ; 0.29)$ & 0.0002 \\
\hline $\begin{array}{l}\text { Protective anti-measels IgG } \\
\text { level, } \mathrm{n}(\%)\end{array}$ & $8(57)$ & $50(68)$ & $40(49)$ & 0.06 \\
\hline $\begin{array}{l}\# \text { anti-parotitis vaccine } \\
\text { doses }\end{array}$ & $2.0(2.0 ; 2.0)$ & $2.0(1.0 ; 2.0)$ & $2.0(1.0 ; 2.0)$ & 0.19 \\
\hline Anti-parotitis IgG, Me/ml & $2.0(1.2 ; 4.3)$ & $2.8(1.3 ; 5.6)$ & $2.5(1.0 ; 5.1)$ & 0.47 \\
\hline $\begin{array}{l}\text { Protective anti-parotitis IgG } \\
\text { level, } \mathrm{n}(\%)\end{array}$ & $12(86)$ & $62(84)$ & $62(76)$ & 0.38 \\
\hline $\begin{array}{l}\# \text { anti-diphtheria vaccine } \\
\text { doses }\end{array}$ & $5.0(4.0 ; 5.0)$ & $4.0(4.0 ; 5.0)$ & $5.0(4.0 ; 5.0)$ & 0.39 \\
\hline Anti-diphtheria IgG, Me/ml & $0.17(0.0 ; 1.2)$ & $0.18(0.0 ; 0.4)$ & $0.1(0.0 ; 0.2)$ & 0.18 \\
\hline $\begin{array}{l}\text { Protective anti-diphtheria } \\
\text { IgG level, } \mathrm{n}(\%)\end{array}$ & $9(64)$ & $42(57)$ & $37(45)$ & 0.22 \\
\hline $\begin{array}{l}\text { \# anti-hepatitis B vaccine } \\
\text { doses }\end{array}$ & $3.0(3.0 ; 3.0)$ & $3.0(3.0 ; 3.0)$ & $3.0(3.0 ; 3.0)$ & 0.91 \\
\hline Anti-hepatitis B IgG, Me/ml & $0.56(0.0 ; 7.5)$ & $11.4(0.3 ; 44.8)$ & $10.0(0.0 ; 44.1)$ & 0.08 \\
\hline $\begin{array}{l}\text { Protective anti-hepatitis B } \\
\text { IgG level, } \mathrm{n}(\%)\end{array}$ & $3(21)$ & $40(54)$ & $42(51)$ & 0.08 \\
\hline \# anti-rubella vaccine doses & $2.0(2.0 ; 2.0)$ & $2.0(1.0 ; 2.0)$ & $2.0(1.0 ; 2.0)$ & 0.19 \\
\hline Anti-rubella IgG, Me/ml & $\begin{array}{c}121.8 \\
(70.0 ; 200.0)\end{array}$ & $\begin{array}{l}95.6 \\
(53.3 ; 198.2)\end{array}$ & $\begin{array}{c}56.4 \\
(37.0 ; 100.1)\end{array}$ & 0.008 \\
\hline $\begin{array}{l}\text { Protective anti-rubella IgG } \\
\text { level, } n(\%)\end{array}$ & $14(100)$ & $73(100)$ & $80(98)$ & 0.34 \\
\hline
\end{tabular}

Conclusion: MTX, biologics and JIA durations are factors influenced on anti-vaccine antibody level. It is necessary to regularly check the levels of anti-vaccine antibodies, especially anti-measels and anti-diphtheria for creation of the individual vaccination plan for JIA patients, treated with MTX and biologics.

Disclosure of Interests: None declared

DOI: 10.1136/annrheumdis-2020-eular.6072

\section{FRI0462 PREDICTIVE FACTORS OF RELAPSE AFTER METHOTREXATE DISCONTINUATION IN JIA PATIENTS WITH INACTIVE DISEASE}

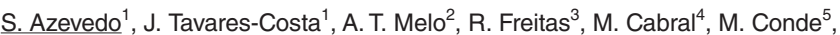
F. Aguiar ${ }^{6}$, A. Neto , A. F. Mourão ${ }^{7}$, F. Oliveira-Ramos ${ }^{2}$, M. J. Santos ${ }^{3}$, D. Peixoto ${ }^{1}$ ${ }^{1}$ Unidade Local de Saúde do Alto Minho, Rheumatology Department, Ponte de Lima, Portugal; ${ }^{2}$ Hospital de Santa Maria, Centro Hospitalar Universitário Lisboa Norte, Pediatric Rheumatology Unit, Lisboa, Portugal; ${ }^{3}$ Hospital Garcia de Orta, Rheumatology Department, Almada, Portugal; ${ }^{4}$ Hospital Prof. Doutor Fernando Fonseca, Pediatric Department, Amadora, Portugal; ${ }^{5}$ Hospital Dona Estefânia, Centro Hospitalar Lisboa Central, Pediatric Department, Lisboa, Portugal; ${ }^{6}$ Centro Hospitalar Universitário São João, Pediatric Rheumatology Unit, Porto, Portugal; ${ }^{7}$ Hospital de Egas Moniz, Centro Hospitalar de Lisboa Ocidental, Rheumatology Department, Lisboa, Portugal

Background: Methotrexate (MTX) is the most widely used conventional synthetic disease-modifying antirheumatic drug (csDMARD) in the treatment of juvenile idiopathic arthritis (JIA). ${ }^{1,2}$ When remission is achieved, questions remain about discontinuing MTX. There is some evidence that a longer period of inactive disease before MTX withdrawal is associated with lower likelihood of relapse, while both rheumatoid factor (RF) positive polyarthritis and extended oligoarthritis categories are associated with higher probability of disease relapse. ${ }^{2,3}$

Objectives: To identify predictive factors of relapse after discontinuation of MTX in JIA patients with inactive disease.

Methods: Prospective multicentre cohort study in patients diagnosed with JIA, according to the ILAR classification, using real world data from the Portuguese national register database, Reuma.pt (Fig 1). ${ }^{4}$ We evaluated patients who have reached JADAS27 inactive disease ( $\leq 1$ and no active extra-articular manifestations) and discontinued MTX before the age of 18 years-old. ${ }^{5}$ Relapse was defined as recurrence ( $>1$ or extra-articular manifestations) or restarting a DMARD. ${ }^{5}$ To identify differences of relapse risk, univariate analyses were performed. Persistence in remission was estimated using the Kaplan-Meier method. Subsequently, Cox regression analyses were performed to identify predictors of relapse.

Results: 119 JIA patients discontinued MTX due to inactive disease (Fig 1). $69.7 \%$ were females and $60.6 \%$ had oligoarticular JIA. Sociodemographic and clinical characteristics are shown in Table 1. Relapse has occurred in $32.8 \%$. Table 2 shows the disease characteristics at MTX initiation and discontinuation and at relapse or last visit.

In univariate analysis, relapse was associated with the use of NSAIDs at the time of MTX discontinuation $(p=.027)$ and with a period of less than two years in inactive disease before MTX suspension $(p=.040)$. We found no association with gender, race, immunology (RF, antinuclear and cyclic citrullinated peptide antibodies), MTX dose, discontinuation modality (tapering and spacing the doses or just tapering the dose), extra-articular manifestations, previous corticotherapy, family history, body mass index, JADAS, CHAQ index, inflammatory parameters, tender and swollen joint counts at MTX initiation or discontinuation nor with age at remission or at MTX suspension. Median persistence in inactive disease was significantly higher in patients with more than two years in remission before MTX discontinuation $(p=.034)$ and in those who did not use NSAIDs at time of MTX discontinuation ( $p=.026$ ) (Fig 2). After adjustment for age at diagnosis, MTX tapering and JIA category, use of NSAIDs at the time of discontinuation ( $\mathrm{HR}, 1.9895 \% \mathrm{Cl}$ 1.03-3.82) and less than two years in remission (HR, $3.1295 \% \mathrm{Cl} 1.35-7.13)$ remained associated with relapse. Conclusion: In this large cohort we found that the use of NSAIDs at the time of MTX discontinuation was associated with two times the likelihood of relapse. Like in other studies we also showed that the time in remission before MTX discontinuation is the main predictor of relapse. We found no association between the JIA category and the risk of relapse.

\section{REFERENCES:}

[1] Hügle B 2016

[2] Klotsche J 2018

[3] Guzman J 2014

[4] Canhão H 2011

[6] Consolaro A 2014
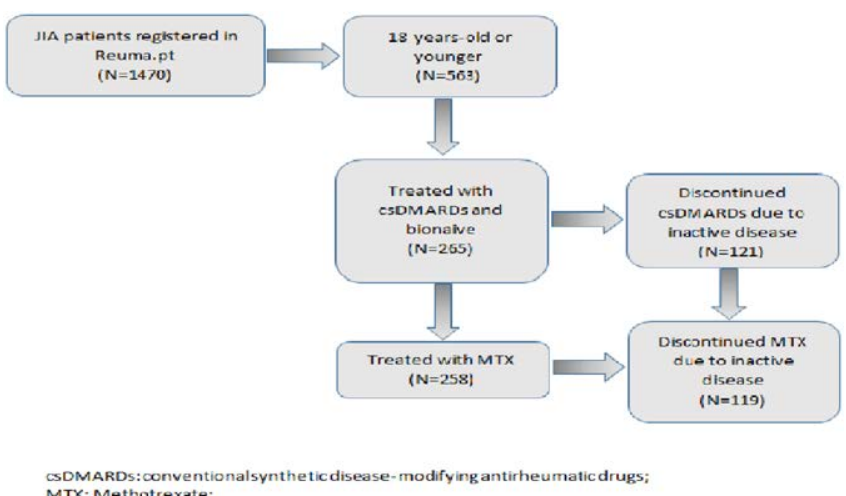
MTX:Methotrexate: Figure 1: Patient flowchart.
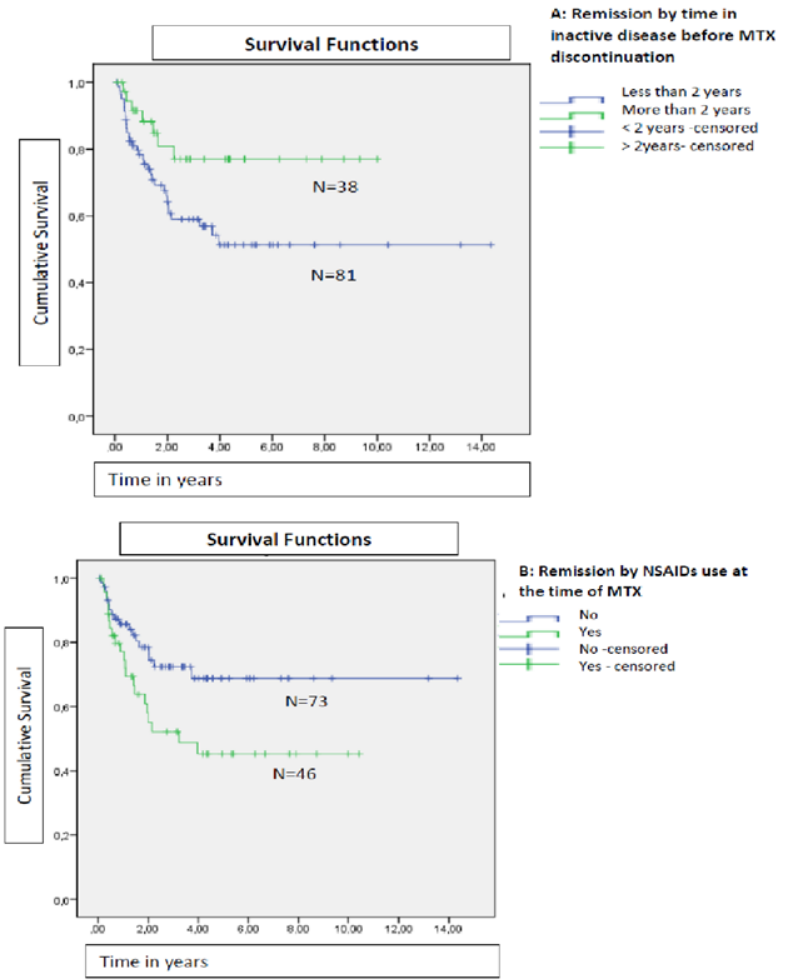

MTX: Methotrexate; NSAIDs: Non-Steroidal Anti-inflammatory Drugs

Figure 2: Kaplan-Meier survival curves showing persistence in remission by A: time in remission hefore $M T X$ discontinuation and $B$. use of NSAPS at the time of MTX discontinuation. 